\title{
Detection and Counting of Red Blood Cells in Blood Cell Images using Hough Transform
}

\author{
Mausumi Maitra \\ Information Technology \\ Govt. College of Engineering \\ and Ceramic Technology \\ 73, A. C. Banerjee Lane, \\ Kolkata, India
}

\author{
Rahul Kumar Gupta \\ Computer Science and \\ Engineering \\ Govt. College of Engineering \\ and Ceramic Technology \\ 73, A. C. Banerjee Lane, \\ Kolkata, India
}

\author{
Manali Mukherjee \\ Information Technology \\ Govt. College of Engineering \\ and Ceramic Technology \\ 73, A. C. Banerjee Lane, \\ Kolkata, India
}

\begin{abstract}
Counting of red blood cells (rbc) in blood cell images is very important to detect as well as to follow the process of treatment of many diseases like anaemia, leukaemia etc. However, locating, identifying and counting of -red blood cells manually are tedious and time-consuming that could be simplified by means of automatic analysis, in which segmentation is a crucial step. In this paper, we present an approach to automatic segmentation and counting of red blood cells in microscopic blood cell images using Hough Transform. Detection and counting of rbc have been done on five microscopic images and finally discussion has been made by comparing the results achieved by the proposed method and the conventional manual counting method.
\end{abstract}

\section{General Terms}

Digital Image Processing

\section{Keywords}

Image Segmentation, Detection, Red Blood Cell, Counting, Hough Transform.

\section{INTRODUCTION}

Content-based image indexing and retrieval has been an important research area in computer science for the last few decades. Many digital images are being captured and stored such as medical images, architectural, advertising, design and fashion images, etc. As a result large image databases are being created and being used in many applications. In this work, the focus of our study is on medical images. A large number of medical images in digital format are generated by hospitals and medical institutions every day. Consequently, how to make use of this huge amount of images effectively becomes a challenging problem [1]. In the field of biomedicine, because of cell's complex nature, it still remains a challenging task to segment cells from its background and count them automatically [2-5]. Counting problem arises in many real world applications including cell counting in microscopic images, monitoring crowds in surveillance systems and performing wildlife census or counting the number of trees in an aerial image of a forest [6-12]. The microscope inspection of blood slides provides important qualitative and quantitative information concerning the presence of hematic pathologies. From decades this operation is performed by experienced operators, which basically performs two main analyses. The first is the qualitative study of the morphology of the cells and it gives information of degenerative and tumoral pathologies. The second approach is quantitative and it consists of differential counting of the blood cells. Automated cell counter systems (for example laser-based citometers [13] are available in the market, but they are not image based or morphological and they destroy the blood samples during the analysis [13,14]. Only few attempts of partial / full automated systems based on image processing systems are present in literature and they are still at prototype stage. Vincenzo et.al. [15] showed the classification and count of white blood cells in microscopic images for the assessment of a wide range of important hematic pathologies.

According to American Cancer Society(2009), the normal red blood cells in our body is divided into four categories of ages, which are new born, children, women and men. Red blood is measured by the amount of hemoglobin. We suffered fatigue and short of breath when the level of hemoglobin is too low due to insufficient oxygen supply. The effect of high red blood cells in our blood can be indication of an undetected heart or lung problems. Therefore, rbc count is very important in diagnosis of many dieses. In this paper we focus on the problem of identification and counting of red blood cells by microscopic images. The proposed work individuates the red blood cells from the other blood cells in the blood cell images by using Hough Transform method and subsequently it counts the number of red blood cells in the images. The whole work has been done on MATLAB 7.1 platform. Finally, the count is normalized to get it per cubic milliliter, which is the normal practice by a medical practitioner.

\section{METHODS}

\subsection{Image Enhancement and Identification of Red Blood Cells}

The purpose of the work is to count the number of red blood cells in a given blood sample. For this we have applied various pre-processing techniques like edge detection, spatial smoothing filtering and adaptive histogram equalization to detect and extract the red blood cells from the images. Feature extraction has been done through the Hough Transform method which has been used to find out the red blood cells based on their sizes and their shapes. This isolates the red blood cells from the rest of the image of the blood sample so that further processes like counting can be applied exclusively on them . Fig.1 shows five blood cell microscopic images taken for our study and fig. 2 represents corresponding pre-processed gray level images. 

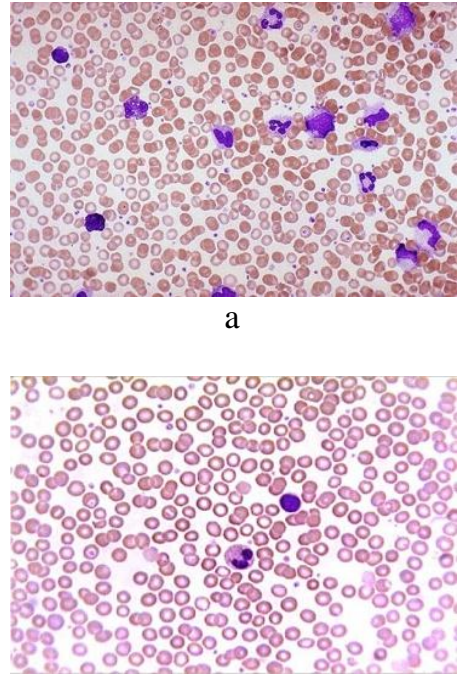

b
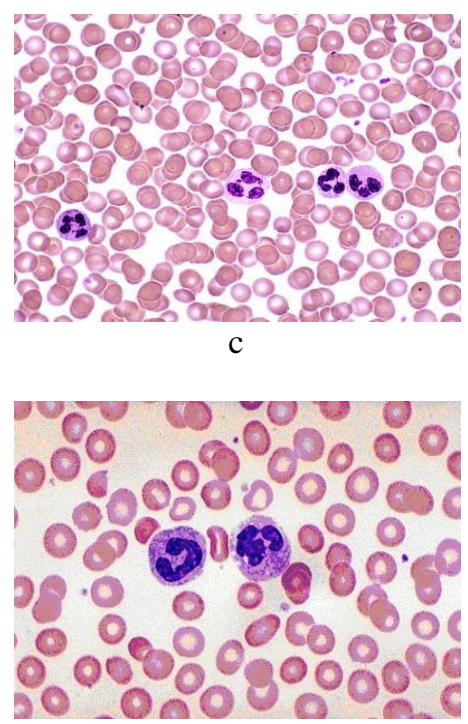

d

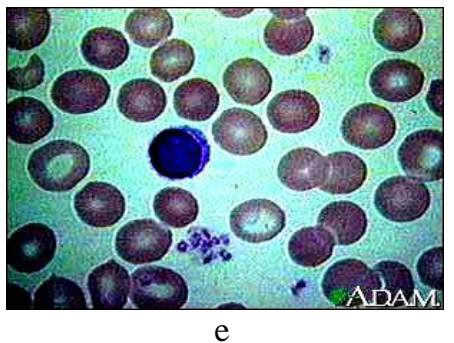

Fig. 1: Original Blood Cell Images

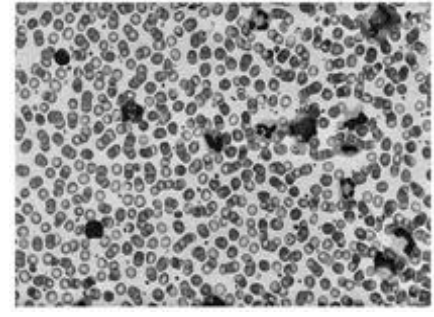

a

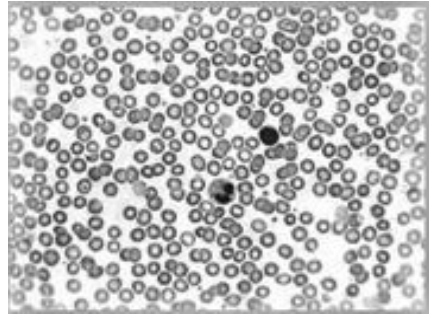

b
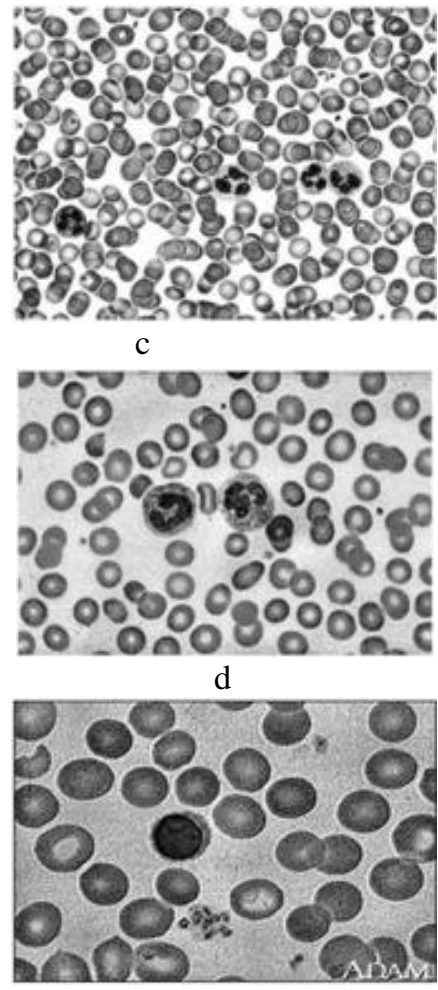

Fig. 2: Pre-processed Gray Level Images

\subsection{Hough Transform in Object Detection}

The Hough transform[16] is a feature extraction technique used in image analysis, computer vision and digital image processing. It was initially suggested as a method for line detection in edge maps of images, then extended to detect general low-parametric objects such as circles $[17,18]$.To detect a straight line in an $\mathrm{n} \times \mathrm{n}$ image, the simplest method is to compute all possible lines defined by every pair of points in the

Rewriting the equation

$$
\mathrm{y}_{\mathrm{i}}=\mathrm{ax}_{\mathrm{i}}+\mathrm{b} \text {, }
$$

$$
b=-a x_{i}+y_{i}
$$

image and then find all subsets of points that are closed to particular line. The computation involved will be enormous because the maximal possible line is $n(n-1) / 2 \sim n^{2}$ and then $(n)$ $[n(n-1)] / 2 \sim n^{3}$. Comparisons need to be performed for each and every point in the image. The problem is solved using Hough Transform that uses the parametric description of the shape to reduce the computation involved. Considering two points $\left(\mathrm{x}_{1}\right.$ , $\left.\mathrm{y}_{1}\right)$ and $\left(\mathrm{x}_{2}, \mathrm{y}_{2}\right)$ in the $\mathrm{x}-\mathrm{y}$ plane, the line equation is:

Two points are represented in the $x-y$ as well as $a-b$ plane. The first point $\left(\mathrm{x}_{1}, \mathrm{y}_{1}\right)$ and the second point $\left(\mathrm{x}_{2}, \mathrm{y}_{2}\right)$ each yield a line in the $\mathrm{a}-\mathrm{b}$ plane and both the lines intersect at a point and this is also true for all the points contained in the line. Using this 
unique feature a parameter space called as the accumulator cell or Hough space is created with a-axis and b-axis having a min and max of the expected range. The same method used for the detection of straight lines can also be extended for the detection of circle and the equation is :

$$
(x-a)^{2}+(y-b)^{2}=r^{2}
$$

The equation for circle detection contains three unknowns (a,b,r) parameters and therefore the accumulator cell should be of three dimensional for three unknown variables.

The purpose of the technique is to find imperfect instances of objects within a certain class of shapes by a voting procedure. This voting procedure is carried out in a parameter space, from which object candidates are obtained as local maxima in a so called accumulator space that is explicitly constructed by the algorithm for computing the Hough Transform. Any Hough Transform based method essentially works by splitting the input image into a set of voting elements. Each such element votes for the hypotheses that might have generated this element. The votes from different voting element pixels are added together into a Hough image, with the height of the peak providing the confidence of the detection.

Hough based approach for object detection is flexible as the primary voting elements are not restricted to edge pixels, but can include interest points [9], image patches [19,20] or image regions[21]. Another attractive property is the simplicity of the learning procedure [7]. Fig. 3 shows the identification of rbcs by Hough Transform.
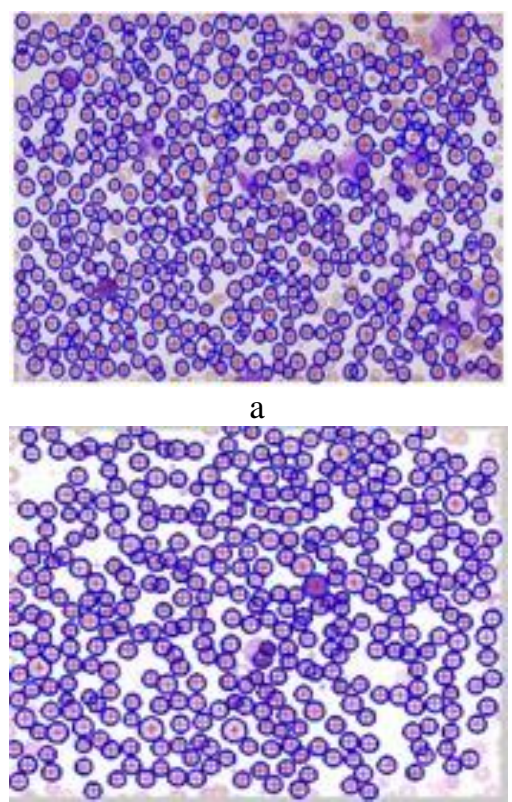

b

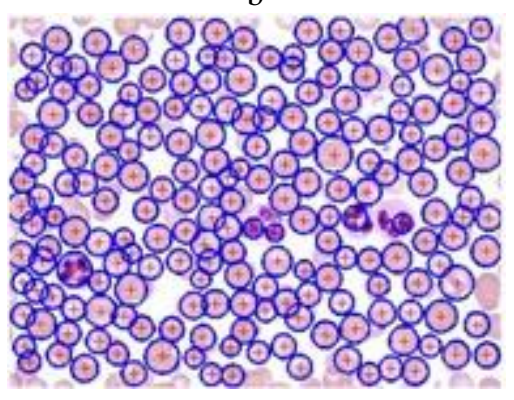

c

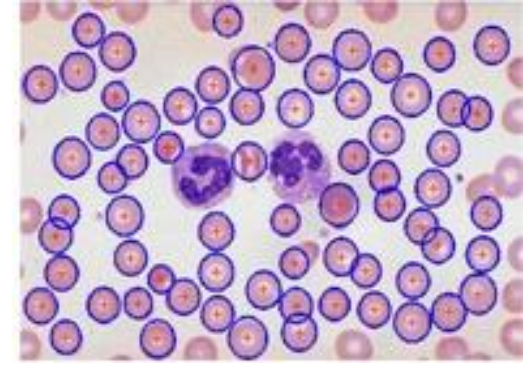

d

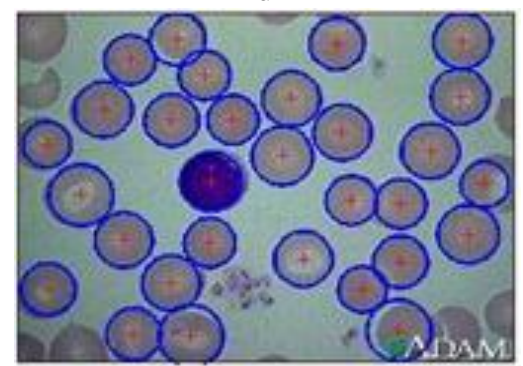

$\mathrm{e}$

Fig. 3: Images after Hough transform

\subsection{Counting}

Having successfully isolated the red blood cells we have applied a counter that has counted the number of rbcs in the image field. However, blood count in medical terms means the number of blood cells ( $r b c$ or wbc or platelets) in a cubic millimeter of blood volume. Hence we have deduced a formula to calculate the number of red blood cells per cumm based on the number of cells in the area of the given image of the blood sample. We have assumed that the thickness of the blood sample film is $0.1 \mathrm{~mm}$ which is the standard medical practice. This allows for an overlapping of maximum two layers in thickness which is the common trend in the images provided in fig.3. This formula requires an input for providing the magnification factor which is the magnification level under the microscope at which the image has been taken. We have taken five blood cell images for our study. Each of the images are preprocessed by the above mentioned techniques. Finally the number of red blood cells were identified and counted in each of these images using Hough Transform. The results of our study have been discussed in Table - 1 along with the different parameters used for each of the images.

\section{RESULTS AND DISCUSSION}

Actual volume of the blood sample is calculated with proper magnification factor ( $\mathrm{X}$ and $\mathrm{Y}$ directions). Now such samples are usually diluted with an anticoagulant liquid to separate the cells to decrease overlapping. In such cases we have to multiply the count by the dilution factor. Considering these factors the formula for rbc count becomes :

Actual rbc count per cumm $=$

(rbc counted by Hough Transform / ((input image area/(magnification $*$ magnification $) *$ film thickness $)) *$ dilution factor 
Table 1. Result of RBC Count by the Proposed Method

\begin{tabular}{|c|c|c|c|c|}
\hline $\begin{array}{l}\text { Image } \\
\text { Samples }\end{array}$ & Magnification & $\begin{array}{l}\text { Radius Range } \\
\text { (in pixel) }\end{array}$ & $\begin{array}{c}\text { Dilution } \\
\text { factor }\end{array}$ & $\begin{array}{c}\text { Blood Count by the proposed method per } \\
\text { cumm(in millions) }\end{array}$ \\
\hline $\mathrm{a}$ & $200 * 200$ & {$[2-14]$} & 10 & 2.38 \\
\hline $\mathrm{b}$ & $300 * 300$ & {$[5-18]$} & 10 & 5.00 \\
\hline $\mathrm{c}$ & $300 * 300$ & {$[5-14]$} & 10 & 3.02 \\
\hline $\mathrm{d}$ & $800 * 800$ & {$[5-25]$} & 10 & 6.22 \\
\hline $\mathrm{e}$ & $1000 * 1000$ & {$[5-18]$} & 10 & 9.12 \\
\hline
\end{tabular}

Table 2. Comparison of the Result between the proposed method and the manual method

\begin{tabular}{|c|c|c|c|}
\hline $\begin{array}{c}\text { Image } \\
\text { Samples }\end{array}$ & $\begin{array}{l}\text { Radius } \\
\text { Range(in } \\
\text { pixels) }\end{array}$ & $\begin{array}{l}\text { Blood } \\
\text { Counted } \\
\text { Manually } \\
\text { per } \\
\text { cumm(in } \\
\text { millions) }\end{array}$ & $\begin{array}{l}\text { Blood } \\
\text { Counted } \\
\text { methodically } \\
\text { per cumm(in } \\
\text { millions) }\end{array}$ \\
\hline a & {$[2-14]$} & 2.80 & 2.38 \\
\hline b & {$[5-18]$} & 5.82 & 5.00 \\
\hline c & {$[5-14]$} & 3.12 & 3.02 \\
\hline d & {$[5-25]$} & 6.40 & 6.22 \\
\hline e & {$[5-18]$} & 9.30 & 9.12 \\
\hline
\end{tabular}

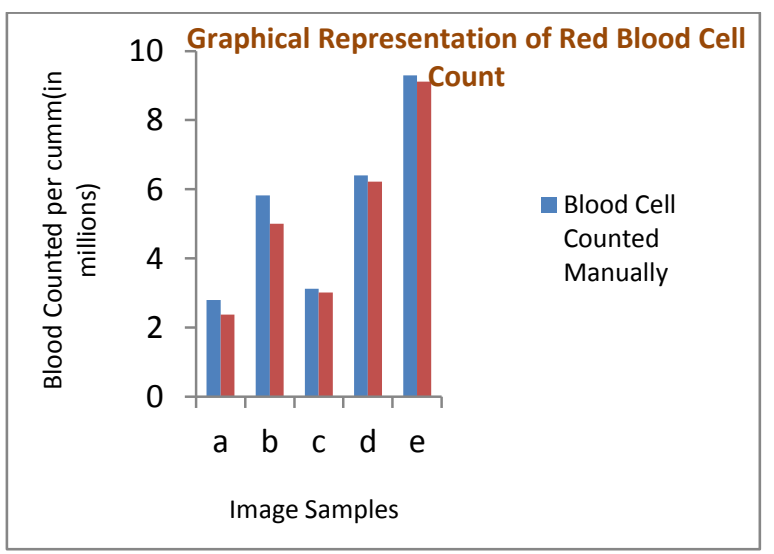

Fig. 4: Graphical Representation of the results

It is observed that the results obtained by the proposed method offer a good conformity with the manual counting method. In our method, we have left out the cells that are not totally in the image field. However, in a real blood test where blood count is done manually, the practice is to count the cells on two adjacent edges of the image field and take each cell as one irrespective of how much of it is in the image field. It is assumed that two opposite sides have same number of such cells. As this edge correction has not been considered, in each of the samples the count values by the proposed method are slightly less than the count values obtained manually. The software must be modified to count those rbcs to obtain more accurate result.

\section{CONCLUSION}

This paper presents a methodology to achieve an automated detection and counting of red blood cells in microscopic images using Hough Transform. Results indicate that the counting of red blood cell in microscopic images offer remarkable accuracy. Laser-based citometers are available to count blood cells, but they are not image based and destroy the blood samples during the analysis. Also installation of such system is very costly. Our proposed method is very cost-effective and can be easily implemented in medical facilities anywhere with minimal investment in infrastructure. It can also identify overlapping blood cells and count them separately. It is also very time effective as manual counting is a very tedious job and time consuming. However, the software must be modified to count the effective number of rbcs which are partly in the image fields to obtain more accurate result.

Further studies will be focused on complete blood cell count i.e. a total count of the number of red blood cells , white blood cells and platelets in the blood sample. This can be easily done by modifying the present software to take into account their different shapes and sizes.

\section{ACKNOWLEDGEMENT}

We acknowledge University Grant Commission (UGC), India, for providing financial support to the authors through the major research project "Development of I P core for Implementing Image Processing Algorithms on FPGA Board" for pursuing this work.

\section{REFERENCES}

[1] Lehmann T.M., Wein B., Dahmen J., Bredno J., Vogelsang F. \& Kohnen M. : Content based image retrieval in medical applications : a novel multi step approach. International Society for Optical Engineering (SPIE), 3972, pp.312-320.(2000)

[2] Dwi Anoragaingrum : Cell segmentation with median filter and mathematical morphology operation, proceeding of the IEEE 10th International Conference on Image Analysis and Processing (ICIAP), pp. 1043-1046 (1999).

[3] Keng Wu et al.: Live cell image segmentation, IEEE Trans on Biomedical Engineering, 42(1), pp.1-12.(1995).

[4] Mark B. Jeacocke, Brian C. Lovell : A Multi-resolution algorithm for Cytological image segmentation, The second Australian and New Zealand conference on intelligent information systems, 322-326 (1994).

[5] Choi H, Baraniuk R., Multiscale : Image segmentation using wavelet-domain hidden Markov models, IEEE Transaction on image processing, 10(9), pp.1309-1321 (2001).

[6] S.Y.Cho, T.W.S.Cho and C.T.Leung, : A neural based crowd estimation by hybrid global learning algorithm, IEEE Transaction on Systems, Man andCybernetics, Part B, 29(4), pp.535-541(1999).

[7] O. Barinova, V.Lempitsky and P. Kohli, : On the detection of multiple object instances using Hough Transforms , CVPR, (2010). 
[8] D. Kong, D. Gray and H. Tao : A viewpoint invariant approach for crowd counting, ICPR (3), pp.1187-1190 (2006)

[9] B. Leibe, A. Leonardis and B. Schiele, Robust object detection with interleaved categorization and segmentation, International journal of Computer Vision, 77(1), 2008, 259-289.

[10] A.N.Marana, S.A.Velastin, L.F.Costa and R.A.Lotufo, Estimation of crowd density using image processing, Image Processing for Security Applications, 1997, 1-8.

[11] D. Ryan, S. Denman, C. Fookes and S. Sridharan : Crowd counting usingmultiple local features, Proceedings of the Digital Image Computing: Techniques and Applications, pp. 81-88 (2009)

[12] V. Lempitsky and A. Zisserman, : Learning to count objects in images, CVPR, NIPS, (CMP Prague Colloquium) (2010).

[13] Abbott Diagnostics Website http://www.abbott.com/products/diagnostics.htm/

[14] Beckman Coulter Website,
[15] Vincenzo Piuri, Fabio Scotti : Morphological classification of blood leucocytes by microscope images, IEEE International conference on Computational Intelligence for Measurement Systems and Applications, pp. 103-108 (2004).

[16] P. V. C. Hough : Method and means of recognizing complex patterns, U. S. patent 3069654, 1962

[17] D. H. Baalard : Generalizing the Hough Transform to detect arbitrary shapes, Pattern Recognition, 13(2), pp.111122 (1981).

[18] R. O. Duda and P. E. Hart : Use of Hough Transformation to detect lines and curves in pictures, Comm. ACM, 15, pp 11-15 (1972).

[19] J.Gall and V.Lempitsky : Class Specific Hough forests for object detection, CVPR, (2009).

[20] R. Okada : Discriminative generalized Hough Transform for object detection, ICCV,( 2009).

[21] C.Gu, J.J.Lim, P. arbelaez and J. Malik : Recognition using regions, CVPR, (2009) http://www.coulter.com/coulter/Hematology/ 